\title{
Enhancement of The Edible Mushroom Pleurotus Ostreatus Yield via A New Liquid Fermentation Tank
}

\author{
Xiaoyu DUAN \\ College of Food Science \\ Sichuan Agricultural University \\ Yaan 625014, China \\ e-mail: 1148037254@qq.com \\ Yuxian YOU \\ College of Food Science \\ Sichuan Agricultural University \\ Yaan 625014, China \\ e-mail: 951814707@qq.com
}

Yiwen LI

College of Food Science

Sichuan Agricultural University

Yaan 625014, China

e-mail: 2510691966@qq.com

\section{Cheng LI}

College of Food Science

Sichuan Agricultural University

Yaan 625014, China

e-mail: lichenglcp@163.com

\author{
Yu HU \\ College of Food Science \\ Sichuan Agricultural University \\ Yaan 625014, China \\ e-mail: 1981278017@qq.com \\ Qianqian TANG \\ College of Food Science \\ Sichuan Agricultural University \\ Yaan 625014, China \\ e-mail: 287381031@qq.com
}

\author{
Lan ZHANG \\ College of Food Science \\ Sichuan Agricultural University \\ Yaan 625014, China \\ e-mail: 1165940683@qq.com
}

\author{
Yuntao LIU* \\ College of Food Science \\ Sichuan Agricultural University \\ Yaan 625014, China \\ e-mail: liuyt@sicau.edu.cn
}

\begin{abstract}
Nowadays, the edible and medicinal values of edible fungi have been widely recognized all over the world, particularly in China and Japan, the edible fungi have become important ingredients in Traditional Chinese Medicine. Hundreds of species of edible fungi had been studied and confirmed with biological activities. Therefore, in order to obtain mycelium with high yield efficiently, this study adopted a new type of edible fungus liquid fermentation tank, with the same culture medium, the mycelial yield and fermentation time of the new tank and the traditional fermentation tank were compared under the same culture condition. The new type of fermentation tank was found to have obvious superiority. Under the fermentation condition of $25{ }^{\circ} \mathrm{C}$ for 7 days, about $200 \mathrm{~g}$ of dry mycelium (fermentation liquid volume: $5 \mathrm{~L}$ ) were produced, which was much larger than that of the traditional fermentation tank. Meanwhile, the fermentation time to produce the same amount of mycelium was discovered to be shorter, in other words, this new type of fermentation tank can make fungi enter the logarithmic growth phase earlier.
\end{abstract}

Keywords-liquid fermentation tank; liquid submerged fermentation; mycelia biomass; fermentation time.

\section{INTRODUCTION}

It is well known that edible fungi are important nutrients, not only for texture and flavour but also for their chemical and medicinal properties, for example, they are lack of energy and fat, but rich in protein, carbohydrate and dietary fiber [1]. Due to the high content of non-starch polysaccharides, edible fungus can be a potential source of dietary fiber [2], with $\beta$-glucose being the most valuable part in fungous cells [3]. In addition, the edible fungi also have anti-cancer, anti-bacterial, anti-viral and anti-tumor activities as well as hypotensive, hypolipidemic, hypoglycemic, immunoregulation function and so on [4-6].

Pleurotus have various biological technologies applications in food, medicine and drug industry, showing a wide range of biological activities [7-8]. It is of great nutritive value with the rich content of essential amino-acid, vitamins and minerals such as calcium, phosphorus and iron [9]. Pleurotus ostreatus fungus, which is classified as a kind of Pleurotus, has been appreciated as an edible and medicinal mushroom. It has been of significant recent interest due to its gastronomic value and nutraceutical properties. Pleurotus ostreatus have a large number of medicinally beneficial 
effects, such as antioxidant, immunomodulatory, antitumor activities and so on [7-8].

The liquid fermentation tank takes advantage of biological fermentation principle to provide the best mycelial nutrients, such as $\mathrm{pH}$, temperature, stirring speed and oxygen supply conditions, making the hypha propagate rapidly to complete a cycle of fermentation in a short period of time. The temperature being too high or low will both impede its cultivation of liquid strains. However, the traditional fermentation tank is not able to control the change of temperature, low degree of intelligentialize makes it impossible to realize remote temperature monitoring. Meanwhile, the structure design of some existing fermentation tank is very unreasonable, their covers are usually fastened by screws, which makes it difficult to install and disassemble. What's worse, the filler is mixed with mechanical agitation, which is prone to bring negative outcomes like being heterogeneous, thus affect the growth of the liquid strains.

Therefore, a new type of edible fungus liquid fermentation tank was adopted in this study. The culture solution in the fermentation tank was stirred and heated by gas produced by air compressor. The gas was heated circularly to avoid the situation of local heating and make the stirring and heating being more homogeneous. The silica gel groove attached to the soffit of the tank cover was buckled on the convex silica gel block attached to the upper surface of the tank cover, and then the tank was fixed with buckles and catching grooves. The installation and disassembly of the cover was successfully simplified and became more convenient.

\section{MATERIAls AND MethodS}

\section{A. General Chemical Reagent}

Chemicals and reagents used in this study were purchased from Chengdu Kelong Chemical Reagent Factory. All chemicals were analytically pure. Distilled water was used throughout the whole experiment.

\section{B. Edible Fungus Liquid Fermentation Tank}

In order to achieve the above purpose, this study adopted the following technical proposal:

- One mechanical mixing ventilation fermentation tank was purchased from Zhucheng Yongxiang Company.

- A group of fermentation experiments came from L. M. Papaspyridi et al. Showed that in a liquid fermentation tank containing $20 \mathrm{~L}$ fermentative broth, about $600 \mathrm{~g}$ dry mycelium can be produced under the optimal conditions of fermentation [7-8].

- A customized edible fungus liquid fermentation tank by our laboratory. This new tank including tank pedestal and bottom, the left side of the surface of the pedestal was equipped with an air compressor, and the right was connected with the tank body through a tank bracket. Air compressor through the exhaust valve connected with the bottom of the tank inlet connection of the exhaust pipe. The upper end of the outer wall of the tank body was orderly provided with an observation mirror, a thermometer and a controller, of which the surface was provided with a touch panel, and the right side was equipped with a pushing hand. The upper surface of the tank body was provided with a silica gel convex block and the outer walls were uniformed with a clamping buckle, left by a rotating shaft to connect with the pot cover. The soffit of the tank body was equipped with a silica gel groove, and the lower end of the outer wall corresponded with buckle groove. And the outer walls of the left and right sides were symmetrical with handle. The controller was connected with an electrically air compressor, an output end of a thermometer, a touch control screen and a wireless module respectively (Fig. 1. The numbers represent the following respects: 1: pedestal; 101: omni-directional wheel; 21: tank bracket; 22: air inlet; 23: observation mirror; 24: thermometer; 25: controller; 26: touch panel; 27: hand slap; 3: air compressor; 31: exhaust valve spindle; 32: exhaust pipe; a: Fermentation tank cover structure; 4 : tank lid.).

\section{Liquid Fermentation}

- The mushroom strains used in this study were derived from our laboratory. They were originally bought in ABa autonomous prefecture in Sichuan province. The gene identification result hasn't been published.

- Pleurotus ostreatus vaccination was inoculated in potato dextrose broth (PDB) medium triangle in the bottle, consists of glucose $20 \mathrm{~g} \cdot \mathrm{L}^{-1}$, peptone $5 \mathrm{~g} \cdot \mathrm{L}^{-}$ ${ }^{1}$, yeast extract $1 \mathrm{~g} \cdot \mathrm{L}^{-1}$, magnesium sulfate $1 \mathrm{~g} \cdot \mathrm{L}^{-1}$, potassium dihydrogen phosphate $1 \mathrm{~g} \cdot \mathrm{L}^{-1}$ and sodium chloride $2 \mathrm{~g} \cdot \mathrm{L}^{-1}[10-11]$.

- Under the condition of 21, 23, 25, 27, 29 degrees centigrade. Culture the mushroom for 7 days, each parameter of four levels.

- Three of the four levels were taken optionally and centrifuged (6000 rpm, 5 minutes and 4 degrees centigrade), then the product were collected.

- The product was lyophilized for $48 \mathrm{~h}$ to obtain dry mycelium.

- Comparing the 15 kinds of experimental data to determine the optimum temperature for the growth of Pleurotus ostreatus.

- The liquid strains were vaccinated in solid medium containing potato dextrose agar (PDA), the culture medium was the same as the second step, and the agar was $12 \mathrm{~g} \cdot \mathrm{L}^{-1}$.

- The agar block containing the equivalent amount of hyphae was transferred to the liquid fermentation tank and cultured for 7 days under optimum temperature.

- The mycelium was collected after centrifugation and lyophilization. 


\section{RESULTS AND DISCUSSION}

According to the preliminary experiment, the optimum temperature of Pleurotus ostreatus was 25 degrees centigrade. Studies show that based on PDA medium, $600 \mathrm{~g}$ of mycelium were obtained after Pleurotus ostreatus were cultured in the traditional fermentation tank containing $20 \mathrm{~L}$ of culture solution, that is to say in $5 \mathrm{~L}$ medium could obtain about $150 \mathrm{~g}$ dry mycelium [7-8]. And about $163 \mathrm{~g}$ dry mycelium can be produced using the purchasable fermentation tank. While using this new type of fermentation tank, about 200 g dry mycelium can be obtained under the same condition. This new type of fermentation tank compared with traditional fermentation tank, mycelia yield increased by $33.3 \%$ and $22.7 \%$, respectively, which has higher efficiency. In addition, three sets of parallel tests proved that it can not only achieve high yield, but also has a high repeatability, which makes a great contribution to the industrial application (Tab. 1, Fig. 2).

Mycelium was collected in 4, 5, 6, 7 days respectively, followed by centrifugation and lyophilization, then the dry weight of mycelia were obtained. According to the data in the figure, in 4.5 days, the efficiency of producing mycelium of the new fermentation tank reached the same level of the traditional one (Tab. 1, Fig. 3). At the same time, we can speculate the logarithmic phase of Pleurotus ostreatus mycelium based on the line chart, which lays a foundation for industrialized production.

In a nutshell, due to its way of stirring and heating gas, this new type of fermentation tank are far higher than that of traditional fermentation tank in both mycelia yield and hypha growth time, suitable for modern production of fungi, also saving a lot of time for the industrial application. To put it simply, it has broad space for development in industrial application.

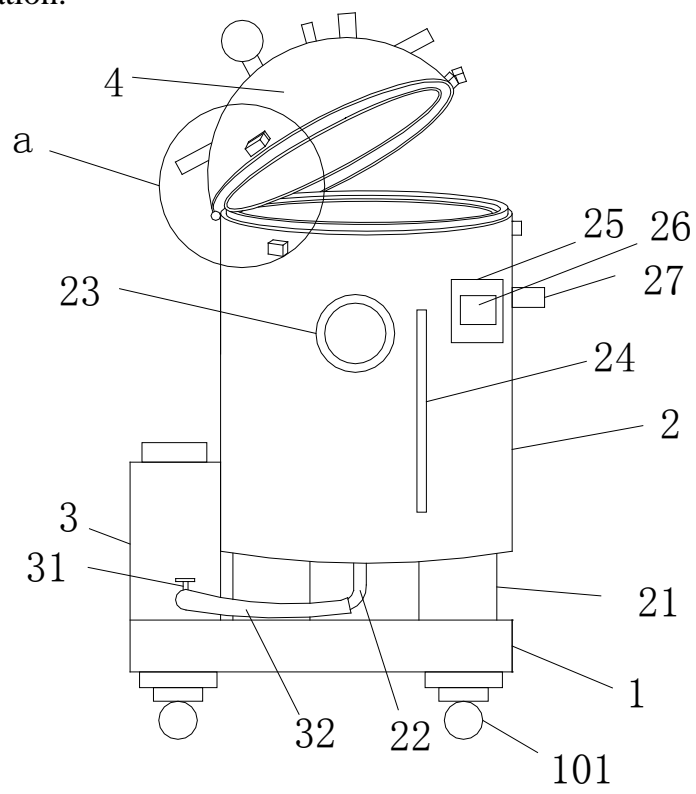

Figure 1 Edible fungus liquid fermentation tank installation drawing
TABLE I MYCELIAL DRY WEIGHT UNDER DIFFERENT CONDITIONS

\begin{tabular}{cccccc}
\hline \multirow{2}{*}{ Time(d) } & \multicolumn{5}{c}{ dry weight of the mycelium(g) } \\
\cline { 2 - 6 } & group 1 & group 2 & group 3 & AVG & SD \\
\hline 4 & 145.0 & 143.8 & 146.8 & 145.2 & 1.23 \\
5 & 171.5 & 168.3 & 172.0 & 170.6 & 1.64 \\
6 & 197.3 & 196.5 & 199.0 & 197.6 & 1.04 \\
7 & 203.0 & 200.8 & 201.3 & 201.7 & 0.94 \\
$7^{*}$ & 154.5 & 170.8 & 166.8 & 164.0 & 6.94 \\
\hline
\end{tabular}

* Stands for the result of use buy fermentation tank.

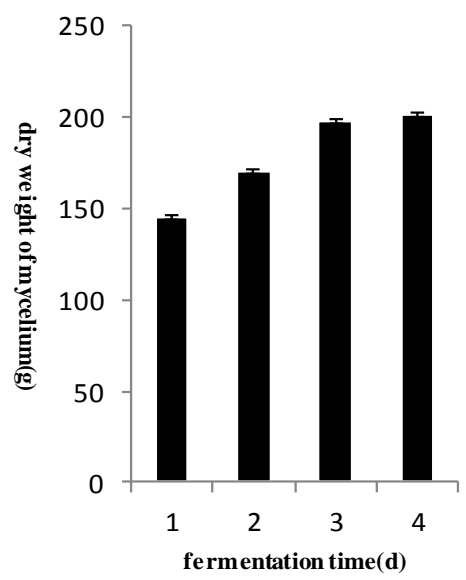

Figure 2 Mycelial day weight under different fermentation time

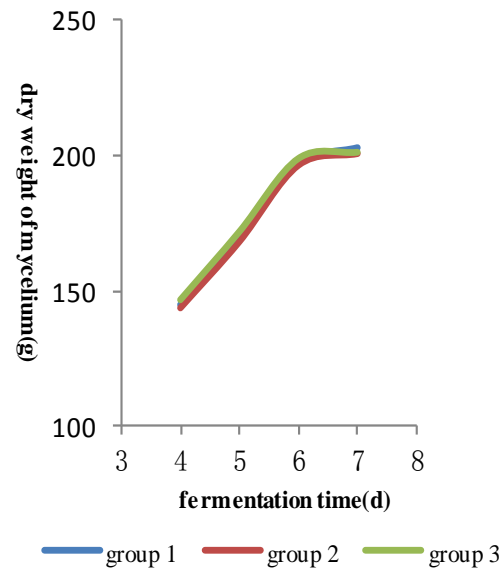

Figure 3 Mycelial growth curve

\section{CONCLUSION}

The Pleurotus ostreatus production of the new type of fermentation tank was significantly superior to the traditional ones. The culture solution was stirred and heated using gas produced by air compressor. With the method of circulating 
gas heating, the mixture can be more uniform and the situation of local heating could be avoided. The amount of hypha produced by traditional fermentation tank was significantly fewer than the new one under the same culture condition, it might cause by the heterogeneous stirring or the insensitive temperature control system, making traditional fermentation tank unsuitable for industrial production. In addition, this new model has convenient for the installation and renovation. What's more, using wireless module connection APP device, which can use electronic equipment to the cultivation of tank temperature and parameters for remote monitoring, make it more intelligent.

\section{ACKNOWLEDGMENT}

This research was financially supported by China Postdoctoral Science Foundation (No. 2015M580795), Fund Project of Sichuan Provincial Department of Education (16ZB0053). X-YD conducted the experiments, performed statistical analysis, and prepared the manuscript. $\mathrm{YH}$ contributed to data interpretation and manuscript preparation. Y-TL contributed to study design, data interpretation, and manuscript preparation. All authors have no conflict of interest to declare.

\section{REFERENCES}

[1] P. Bhatiaa, F.Aurelib, M.D. Amato et al, "Selenium Bioaccessibility and Speciation in Biofortified Pleurotus Mushrooms Grown on Selenium-rich Agricultural Residues,” Food Chemistry, vol. 140, Sep. 2013, pp. 225-230, doi: 10.1016/ j.foodchem.2013.02.054.

[2] S. K. Singdevsachan, P. Auroshree, J. Mishra et al, "Mushroom Polysaccharides as Potential Prebiotics with Their Antitumor and Immunomodulating Properties: A review," Bioactive carbohydrates and dietary fibre, vol. 7, Nov. 2015, pp. 1-14, doi: 10.1016/j.bcdf.2015.11.001.

[3] Chi-Hsein Chao, Hui-Ju Wu, Mei-Kuang Lu, "Promotion of Fungal Growth and Underlying Physiochemical Changes of Polysaccharides in Rigidoporus ulmarius, an Edible Basidiomycete Mushroom," Carbohydrate Polymers, vol. 85, Mar. 2011, pp. 609-614, doi:10.1016/j.carbpol.2011.03.023.
[4] Jianjun Zhang, Min Liu, Yongheng Yang et al, "Purification, Characterization and Hepatoprotective Activities of Mycelia Zinc Polysaccharides by Pleurotus djamor, "Carbohydrate Polymers, vol. 136, Sep. 2015, pp. 588-597, .doi /10.1016/j.carbpol.2015.09.075

[5] Yiwen Li, Yuxian You, Qianqian Tang et al, "Preparation of Carboxymethylated Polysaccharides from Mycelia of Catathelasma ventricosum and Their Antidiabetic Properties,” 3rd International Conference on Materials Engineering, Manufacturing Technology and Control (ICMEMTC 2016), Atlantis Press, 2016, pp. 73-80, doi: 10.2991/icmemtc-16.2016.15

[6] J. Hamuro, Y. Tamashita, Y. Ohaska, Y.Y. Maeda and G. Chihara, "Carboxymethylpachymaran, a New Water Soluble Polysaccharide with Marked Antitumour Activity,” nature, vol. 233, Oct. 1971, pp.486-488, doi:10.1038/nature. 233486a0.

[7] L. M. Papaspyridi, N. Aligiannis, P. Christakopoulos, A.L. Skaltsounis, N. Fokialakis, "Production of Bioactive Metabolites with Pharmaceutical and Nutraceutical Interest by Aubmerged Fermentation of Pleurotus ostreatus in a Batch Atirred Tank Bioreactor," Selection and/or peer-review under responsibility of 11th International Congress on Engineering and Food (ICEF 11) Executive Committee, Elsevier B.V, Sep. 2011, pp. 1746-1752, doi:10.1016/j.profoo.2011.09.257.

[8] L.M. Papaspyridi, N. Aligiannis, E. Topakas et al, "Submerged Fermentation of the Edible Mushroom Pleurotus ostreatus in a Batch Stirred Tank Bioreactor as a Promising Alternative for the Effective Production of Bioactive Metabolites,” molecules, vol. 17, Mar. 2012, pp. 2714-2724, doi:10.3390/molecules17032714.

[9] Marliane C.S. da Silva, J. Naozuka , José Maria R. da Luz et al, "Enrichment of Pleurotus ostreatus Mushrooms with Selenium in Coffee Husks,” Food Chemistry, vol. 131, Mar 2012, pp. 558-563, doi:10.101/j.foodchem.2011.09.023.

[10] Shan Lin, Zhi-Qiang Liu, Peter James Baker, "Enhancement of Cordyceps Polysaccharide Production via Biosynthetic Pathway Analysis in Hirsutella sinensis," International Journal of Biologica Macromolecules, vol. 92, Aug. 2016, pp.872-880, doi: 10.1016/j.ijbiomac.2016.08.002.

[11] N. Golan-Rozen, B. Seiwert, C. Riemenschneider, "Transformation Pathways of the Recalcitrant Pharmaceutical Compound Carbamazepine by the White-Rot Fungus Pleurotus ostreatus: Effects of Growth Conditions,” Environmental Science \& Technology, vol. 49, Sep. 2015, pp. 12351-12362, doi: 10.1021/acs.est.5b02222. 